\title{
Exactly solvable tight-binding model on the RAN: fractal energy spectrum and Bose-Einstein condensation
}

\author{
Maurizio Serva ${ }^{1,2}$ \\ ${ }^{1}$ Departamento de Biofísica e Farmacologia, Universidade Federal do Rio Grande do Norte, 59072-970, Natal-RN, Brazil \\ ${ }^{2}$ Dipartimento di Ingegneria e Scienze dell'Informazione e Matematica, Università dell'Aquila, 67010 L'Aquila, Italy
}

(Dated: April 20, 2022)

\begin{abstract}
We initially consider a single-particle tight-binding model on the Regularized Apollonian Network (RAN). The RAN is defined starting from a tetrahedral structure with four nodes all connected (generation 0). At any successive generations, new nodes are added and connected with the surrounding three nodes. As a result, a power-law cumulative distribution of connectivity $P(k) \propto 1 / k^{\eta}$ with $\eta=\ln (3) / \ln (2) \approx 1.585$ is obtained.
\end{abstract}

The eigenvalues of the Hamiltonian are exactly computed by a recursive approach for any size of the network. In the infinite size limit, the density of states and the cumulative distribution of states (integrated density of states) are also exactly determined. The relevant scaling behavior of the cumulative distribution close to the band bottom is shown to be power law with an exponent depending on the spectral dimension and not on the embedding dimension.

We then consider a gas made by an infinite number of non-interacting bosons each of them described by the tight-binding Hamiltonian on the RAN and we prove that, for sufficiently large bosonic density and sufficiently small temperature, a macroscopic fraction of the particles occupy the lowest single-particle energy state forming the Bose-Einstein condensate. We determine no only the transition temperature as a function of the bosonic density, but also the fraction of condensed particle, the fugacity, the energy and the specific heat for any temperature and bosonic density.

PACS numbers: 05.30.Jp, 67.85.Jk, 64.60.aq, 64.60.F-

\section{INTRODUCTION}

The Bose-Einstein condensation (BEC) is one of the most intriguing quantum phenomena involving the formation of a collective quantum state by a gas of identical non-interacting or weakly-interacting bosons. Below transition temperature, the collective state emerges as a consequence of the fact that a macroscopic fraction of the particles occupy the lowest single-particle energy state. For many decades BEC has remained in the realm of theoretical predictions. Only recently it received an impressive experimental demonstration which, in turn, has stimulated a new wealth of theoretical work.

The experimental realization of Bose-Einstein condensate was first obtained for weakly-interacting low-temperature atoms in a magnetic trap [1, 2] proving that BEC is a purely quantum phenomenon that can take place even in absence of inter-particle interactions. More recently, BEC has also been reported in solid state quasi-particles systems such as excitons, antiferro and ferromagnetic magnons, even at room temperature $3-7$.

The collective state emerges if the particle density is sufficiently high and the temperature is sufficiently low. Dimension is also important; in fact, it is well known that free bosons hopping on translationally invariant networks cannot undergo Bose-Einstein condensation if the space dimension $d$ is less than or equal to 2. Recent studies suggest the possibility that the network topology may act as a catalyst for Bose-Einstein condensation allowing condensation even if $d \leq 2$. The presence of BEC has been, in fact, proven, for simple $d \leq 2$ non-translationally invariant networks as comb, star and wheel lattices [8], as well as in complex networks as the Apollonian one 14 17.

To add more evidence to the catalyst role of topology, we shall investigate in this work the properties of noninteracting bosons hopping on the Regularized Apollonian Network, showing that this topology implies macroscopic occupation of the ground state at low temperatures.

The RAN [18, 19], as well its original unregularized version (AN) 20, has a complex architecture characterized by a power-law distributed connectivity (scale free) and an average minimal path between two nodes smaller than any power of the system size (small-world effect). These two properties, which are shared by most of real world networks, make Apollonian Networks an intriguing substrate for statistical models of physical and biological phenomena, as BEC [14 17], the spreading of epidemics [21, 22], the democratic majority vote [23], Ising systems [18, 19, 24, 25], Potts systems [26], disk packing [27] and even gossip spreading [28].

In this paper we consider a tight-binding model on the RAN, whose Hamiltonian has non-vanishing hopping energies only between connected nodes. The Hamiltonian eigenvalues are achieved by a recursive approach for any size of the network. The regularization of the network is essential since it removes the asymmetry associated to the corners, allowing for exact iterative calculations which can be done only approximatively using AN [17. In the infinite size limit, the density of states and the cumulative distribution of states (integrated density of states) are also exactly 
determined. The relevant scaling behavior of the cumulative distribution close to the band bottom is shown to be power law, with an exponent depending on the spectral dimension and not on the embedding dimension.

We then consider a gas made by an infinite number of non-interacting bosons each of them described by the tight-binding Hamiltonian on the RAN and we prove that for sufficiently large bosonic density and sufficiently small temperature a macroscopic fraction of the particles occupy the lowest single-particle energy state forming the BoseEinstein condensate. We determine not only the transition temperature as a function of the bosonic density, but also the fraction of condensed particles, the fugacity, the energy and the specific heat for any temperature and bosonic density.

The paper is organized as follows: in section 2 we describe the RAN model, together with its main properties, including those which differ with respect to AN and which are relevant for exact computing. Then, in section 3, we define the tight-binding Hamiltonian of the single particle and we exactly compute its eigenvalues for any finite size of the network. The density of state for the infinite size network, as well as the cumulative distribution of states are computed in section 4, were we also describe the properties of the cumulative distribution in the relevant region close to the band bottom. Section 5 is devoted to the description of the thermodynamics of a system composed by an infinite number of identical bosons, proving the phase-transition occurrence and finding the transition line in the density/temperature plane. Moreover we determine the fraction of condensed particle, the fugacity, the energy and the specific heat for any temperature and bosonic density. Finally, our main achievements are summarized in section 6 .

\section{REGULARIZED APOLLONIAN NETWORK (RAN)}

The geometrical construction of the Regularized Apollonian Network (RAN) can be done iteratively by initially taking a $g=0$ generation network with 4 nodes all connected, forming a tetrahedral structure with 6 bonds (Fig. 1, left picture). Each of the 4 triples of nodes individuates a different elementary triangle. At generation $g=1$ a new node is added inside each of the 4 elementary triangles and it is connected with the surrounding 3 nodes, so that the network has 8 nodes, 18 bonds and 12 elementary triangles (Fig. 1, right picture). Then the procedure is iterated at any successive generation inserting new nodes in the last created elementary triangles, and connecting each of them with the three surrounding nodes. As a result, one has the following properties for a network of generation $g$ :

- the number of new nodes created at any generation $g^{\prime}$ with $1 \leq g^{\prime} \leq g$ is $V_{g^{\prime}}=4 \times 3^{g^{\prime}-1}$;

- the total number of nodes at generation $g \geq 0$ is $N_{g}=4+\sum_{g^{\prime}=1}^{g} 4 \times 3^{g^{\prime}-1}=2 \times 3^{g}+2$.

The following asymptotic relations holds for large value of g: the total number of nodes is $N_{g} \simeq 2 \times 3^{g} \simeq 3 N_{g-1}$; the number of new nodes created at generation $g$ is $V_{g} \simeq(2 / 3) N_{g}$ (the symbol $\simeq$ indicates in this paper that the ratio of the two sides tends to 1 , the symbol $\sim$ indicates the weaker statement that the ratio tends to a strictly positive constant, finally, the symbol $\approx$ will be used for generic approximate equalities).

The connectivity of a node is defined as the number of connections to other nodes. In $R A N$ the connectivity of each of the already existing nodes (the so-called old nodes) is doubled when generation is updated, while the connectivity of the newly created nodes (the new nodes) always equals 3, leading to the following relevant property:

- the connectivity at generation $g$ of a node $i$ only depends on its age. More explicitly, its connectivity is $3 \times 2^{g-g_{i}^{\prime}}$ where $g_{i}^{\prime}$ is the generation at which it was created, and $n_{i}=g-g_{i}^{\prime}$ is its age. Also, one half of the connections of each old node are with new nodes.

This property, which is crucial for our exact solution via renormalization, is not shared by AN. In fact, in AN the connectivity depends both on age and geometry since the three nodes at the three external corners have a variant connectivity.

The characterization of the RAN can be completed by a description of the connectivity distribution, which can be straightforwardly deduced by the three previously listed properties:

- the number of nodes having connectivity $k$ is $m(k, g)$ which equals $4 \times 3^{g-g^{\prime}-1}$ if $k=3 \times 2^{g^{\prime}}$ with $g^{\prime}=0, \ldots ., g-1$, equals 4 if $k=3 \times 2^{g}$, and equals 0 otherwise.

Accordingly, the cumulative distribution of connectivity $P(k)$ is:

- $P(k)=\sum_{r^{\prime} \geq k} m\left(k^{\prime}, g\right) / N_{g}$ which, for large values of $g$, exhibits a power-law behavior i.e., $P(k) \propto 1 / k^{\eta}$, with $\eta=\ln (3) / \ln (2) \approx 1.585$, 
from which the average connectivity can be simply computed and it is $\simeq 6$, where the approximation holds for large $g$.

The power law cumulative distribution of connectivity $P(k)$ is shared with the AN as well. Analogously to AN, RAN is scale-free and, as already mentioned, it displays the small word effect.
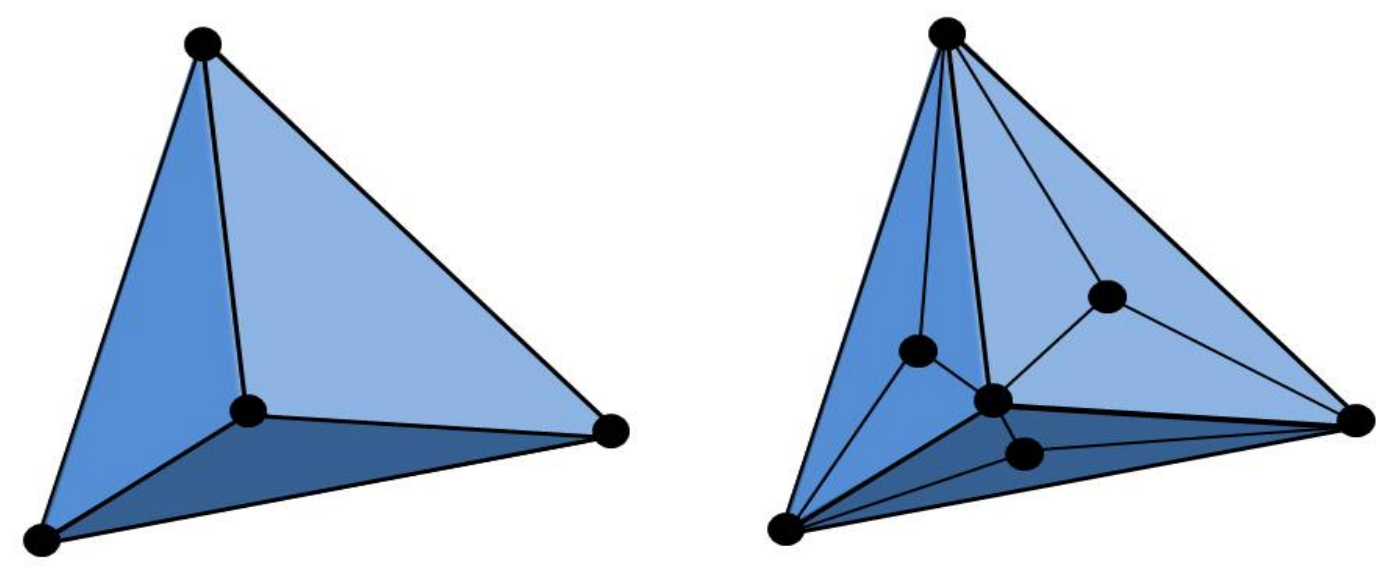

FIG. 1: RAN at generation $g=0$ has 4 nodes all connected, forming a tetrahedral structure with 6 bonds (left picture). Each of the 4 triples of nodes individuates a different elementary triangle. At generation $g=1$ a new node is added inside each of the 4 elementary triangles, and it is connected with the surrounding 3 nodes, so that the network has 8 nodes, 18 bonds and 12 elementary triangles (right picture). The procedure is then iterated at any successive generation inserting new nodes in the last created elementary triangles, and connecting each of them with the three surrounding ones.

We stress once more that the reason for regularizing is that in RAN, at variance with AN, the connectivity of the nodes only depends on their age. The removal of the asymmetry associated to the corners allows for exact iterative calculations, while in AN they can be done only approximatively [17.

\section{TIGHT-BINDING HAMILTONIAN ON THE RAN}

\section{A. The model}

We consider a tight-binding single-particle Hamiltonian on the RAN network. Each node of the network will be considered as having a single orbital, whose on node energy will be assumed vanishing without loss of generality. Moreover, only directly connected nodes hopping amplitudes are included. We represent with $|i\rangle$ the state where the particle is localized at node $i$. Then, the hopping amplitudes $h_{i, j}$ are non-null only between connected nodes of the network; as they are real one has $h_{i, j}=h_{j, i}$. Accordingly, the Hamiltonian assumes the form

$$
H_{g}=\sum_{i, j} h_{i, j}|i\rangle\langle j|,
$$

where the sum goes on all the (ordered) connected pairs $i, j$ of nodes of RAN of generation $g$.

The most general class of models which comply with the symmetry of RAN is achieved assuming that the hopping amplitudes $h_{i j}$ depend on the connectivities of the involved nodes. In other words $h_{i, j}=h\left(k_{i}, k_{j}\right)$. Since $k_{i}=3 \times 2^{n_{i}}$, 
where $n_{i}=g_{i}-g_{i}^{\prime}$ is the age of node $i$, in RAN, the connectivity dependence is the same as age dependence. This implies that we can also rewrite $h_{i, j}=h\left(n_{i}, n_{j}\right)$.

In this work we will assume that the hopping energies are $h_{i, j}=t / \sqrt{k_{i} k_{j}}$. The positive parameter $t$ is the only relevant microscopic energy scale and, from a computational point of view, it can be chosen without loss of generality equal to 1 ( for $t \neq 1$, all eigenvalues are simply multiplied by $t$ ).

The off-diagonal Hamiltonian elements $h_{i, j}$ are inversely proportional to the square root of the product of the connectivity of the two nodes. This rescaling avoids the divergence of the ground state energy, while keeping the energy bandwidth finite in the infinite size limit of the network $(g \rightarrow \infty)$. Rescaling of the microscopic energy scale is commonly used for systems presenting high connectivity as mean-field models or models with long-range connections (see for example Refs. [29 33] ) in order to keep the physical quantities well defined when the size diverges.

\section{B. Eigenvalues}

In this section we compute the eigenvalues of the Hamiltonian $H_{g}$ for any $g$. The number of eigenvalues equals the number of nodes and, therefore, it is $N_{g}=2 \times 3^{g}+2$.

Consider a RAN of generation $g$ on this network and consider an old node (a node that is not newly created at last generation $g$ ). Let us call this node 0 and let us consider all its $k_{0}$ connected nodes labeled by $i \quad\left(i=1,2, \ldots, k_{0}=3 \times 2^{n_{0}}\right.$ where $n_{0}$ is the age of node 0 ); the local Schrödinger equation centered over node 0, correspondingly to an eigenvalue $\epsilon$, reads

$$
\epsilon \psi_{0}=\sum_{i=1}^{k_{0}} h_{0, i} \psi_{i}
$$

where $k_{0}$ is even and among the $k_{0}$ connected nodes there are $k_{0} / 2$ newly created nodes at generation $g$ and $k_{0} / 2$ older nodes. Let us use the even index $2 i=2,4, \ldots ., k_{0}$ for the newly created and an odd index $2 i-1=1,3, \ldots ., k_{0}-1$ for the others.

Let us now consider the $k_{0} / 2$ local Schrödinger equations centered over newly created nodes connected to node 0 . We have

$$
\epsilon \psi_{2 i}=h_{2 i, 0} \psi_{0}+h_{2 i, 2 i-1} \psi_{2 i-1}+h_{2 i, 2 i+1} \psi_{2 i+1}
$$

where we use the convention $\psi_{k_{0}+1}=\psi_{1}$ and $h_{k_{0}, k_{0}+1}=h_{k_{0}, 1}$.

Assume that $\epsilon \neq 0$. In this case (and only in this case) the $\psi_{2 i}$ can be integrated, i.e. they can be substituted from equation (3) into equation (2). In this way, we obtain an equation which connects node 0 with nodes with an odd index, i.e. an equation which only concerns those $k_{0} / 2$ older nodes already existing at generation $g-1$. Then, taking into account that all $h_{0,2 i}$ are equal and $h_{0,2 i}=h_{2 i, 0}=1 / \sqrt{3 k_{0}}$, and considering also that $h_{0,2 i} h_{2 i, 2 i-1}=\frac{1}{3} h_{0,2 i-1}$ we obtain

$$
\left(\epsilon-\frac{1}{6 \epsilon}\right) \psi_{0}=\sum_{i=1}^{k_{0} / 2}\left(1+\frac{2}{3 \epsilon}\right) h_{0,2 i-1} \psi_{2 i-1} .
$$

The above equation, provided that $\epsilon \neq 0$, holds at any of the nodes which were already present at generation $g-1$ and, therefore, it can be compared with the local Schrödinger equation centered on node 0 at generation $g-1$

$$
\epsilon^{\prime} \psi_{0}=\sum_{i=1}^{k_{0} / 2} h_{0,2 i-1}^{\prime} \psi_{2 i-1}
$$

Since at generation $g-1$ all connectivities are one half of connectivities of generation $g$, the hopping energies $h_{0,2 i-1}^{\prime}$ at generation $g-1$ are twice the hopping energies $h_{0,2 i-1}$ of generation $g$. Then the two equations coincide provided that

$$
2\left(\epsilon-\frac{1}{6 \epsilon}\right)=\epsilon^{\prime}\left(1+\frac{2}{3 \epsilon}\right)
$$

which proves the following: a non vanishing $\epsilon$ is an eigenvalue of the Hamiltonian $H_{g}$ if and only if $\epsilon^{\prime}$ is an eigenvalue of the Hamiltonian $H_{g-1}$. 
Eq. (6) can be solved with respect to $\epsilon$ giving immediately:

$$
\epsilon_{1,2}=\frac{1}{4}\left(\epsilon^{\prime} \pm \sqrt{\left(\epsilon^{\prime}\right)^{2}+\frac{8}{3}\left(2 \epsilon^{\prime}+1\right)}\right) .
$$

As we have assumed that $\epsilon \neq 0$, equation (7) only gives the non vanishing eigenvalues of generation $g$, once we know the eigenvalues (vanishing and non vanishing) of generation $g-1$. If there are missing eigenvalues after using (7), they must be vanishing eigenvalues. Therefore, the $N_{g}$ eigenvalues of the generation $g$ Hamiltonian can be generated applying (7) to all $N_{g-1}$ eigenvalues of the generation $g-1$ Hamiltonian. In this way $2 N_{g-1}$ eigenvalues are generated but only those which are not vanishing are retained. Then vanishing eigenvalues are added in order that the total number is $N_{g}$.

Since $N_{g} \geq 2 N_{g-1}$ (the equality only holds when $g=1$ while for large $g$ one has $N_{g} \simeq 3 N_{g-1}$ ), we can simplify as follows:

(i) equation (7) is applied to all $N_{g-1}=2 \times 3^{g-1}+2$ eigenvalues of the generation $g-1$ Hamiltonian. In this way $2 N_{g-1}=4 \times 3^{g-1}+4$ eigenvalues are generated and all retained (including those which are null),

(ii) $N_{g}-2 N_{g-1}=2 \times 3^{g-1}-2$ vanishing eigenvalues are added in order that the total number is $N_{g}=2 \times 3^{g}+2$.

When $g=0$, the RAN reduces to four nodes which are all connected so that the connectivity equals 3 for all nodes and all the hopping amplitudes equal 1/3. Thus, the Hamiltonian (1) can be written as a matrix, yielding

$$
H_{0}=\frac{1}{3}\left[\begin{array}{llll}
0 & 1 & 1 & 1 \\
1 & 0 & 1 & 1 \\
1 & 1 & 0 & 1 \\
1 & 1 & 1 & 0
\end{array}\right]
$$

which has eigenvalues $1,-1 / 3,-1 / 3,-1 / 3$. Then, the eigenvalues of $H_{g}$ can be iteratively obtained applying the rules (i) and (ii). Since for large $g$ the number of vanishing eigenvalues is $N_{g}-2 N_{g-1} \simeq N_{g} / 3$, about $1 / 3$ of vanishing are added at any generation updating.

We remark that equation (7) maps real numbers in the interval $[-1 / 2,1]$ into real numbers in the same interval. Therefore, since the the four eigenvalues corresponding to $g=0$ are in this range, and at any generation only vanishing eigenvalues can be added, the density of states must have a support in $[-1 / 2,1]$. It can be also easily verified that at any generation $g>0$ both a single maximum eigenvalue $\epsilon_{\max }=1$ and a single minimum eigenvalue $\epsilon_{0}=-1 / 2$ are present.

We also remark that $\epsilon_{1,2}$ are always distinct since the argument of the square root in (7) never vanishes in the region $[-1 / 2,1]$. On the other hand, reversing (7) we have that $\epsilon^{\prime}=\left(6 \epsilon^{2}-1\right) /(3 \epsilon+2)$ so that the same $\epsilon$ cannot be associated to two ore more different $\epsilon^{\prime}$. Moreover, one vanishing eigenvalue $\epsilon$ is created by (7) from $\epsilon^{\prime}=-1 / 2$ excluding the first iteration from generation $g=0$ to generation $g=1$. Finally, the number of distinct eigenvalues at generation $g=0$ is 2 and at generation $g=1$ is 4 . In conclusion, the number $L_{g+1}$ of distinct eigenvalues at generation $g+1$ exactly satisfies $L_{g+1}=2 \times L_{g}$.

Summing up, $L_{g}=2^{g+1} \sim N_{g}^{\ln (2) / \ln (3)}$ where $N_{g}=2 \times 3^{g}+2$ is the total number of eigenvalues so that the fractal dimension of the support of the density of states is $\nu=1 / \eta=\ln (2) / \ln (3) \approx 0.631$ ( $\eta$ is the exponent in the cumulative distribution of connectivity).

Before concluding this section we would like to stress that analogous calculations were already made in [17 for AN but only approximately or suitably modifying the Hamiltonian. On the contrary, all calculations in this section using RAN, are exact as well those contained in next sections.

\section{DENSITY OF STATES IN THE INFINITE SIZE LIMIT.}

We have seen that for large $g$ the number of eigenvalues of generation $g-1$ is about $N_{g-1} \simeq 2 \times 3^{g-1}$, while the number of eigenvalues of generation $g$ is about $N_{g} \simeq 2 \times 3^{g} \simeq 3 N_{g-1}$. The iterating procedure produces about $2 N_{g-1}$ non-vanishing eigenvalues of generation $g$ from the eigenvalues of generation $g-1$ by (8). Therefore, about $N_{g-1}$ vanishing eigenvalues must be added in order to complete the list of the $N_{g} \simeq 3 N_{g-1}$ eigenvalues of generation $g$.

For large $g$, the weight of of vanishing eigenvalues is always $1 / 3$ while, at any iteration of equation (8), the weight of any new eigenvalues $\epsilon$ is $1 / 3$ of the weight of the corresponding eigenvalue $\epsilon^{\prime}$. Thus, it is straightforward to derive the density of states 10 which holds in the infinite size limit $g \rightarrow \infty$ : 
- Consider the sequence $\bar{\sigma}_{k}=\sigma_{1}, \sigma_{2}, \ldots \ldots, \sigma_{k}$ of dichotomous variables $\sigma_{i}= \pm 1$ and define iteratively

$$
\epsilon\left(k+1, \bar{\sigma}_{k+1}\right)=\frac{1}{4}\left(\epsilon\left(k, \bar{\sigma}_{k}\right)+\sigma_{k+1} \sqrt{\left[\epsilon\left(k, \bar{\sigma}_{k}\right)\right]^{2}+\frac{8}{3}\left(2 \epsilon\left(k, \bar{\sigma}_{k}\right)+1\right)}\right)
$$

with $\epsilon(0)=0$. One has $\epsilon\left(1, \bar{\sigma}_{1}\right)=\sigma_{1} / \sqrt{6}$ and, in general, the variables $\epsilon(k, \bar{\sigma})$ only depends on the sequence $\sigma_{1}, \sigma_{2}, \ldots \ldots, \sigma_{k}$ so that for any $k$ they are $2^{k}$.

- The weight of a given $\epsilon(k, \bar{\sigma})$ equals $(1 / 3)^{k+1}$ independently on $\bar{\sigma}$. Then, the density of states in the $g \rightarrow \infty$ limit is straightforwardly given by

$$
\rho(\epsilon)=\sum_{k=0}^{\infty}(1 / 3)^{k+1} \sum_{\bar{\sigma}_{k}} \delta\left(\epsilon-\epsilon\left(k, \bar{\sigma}_{k}\right)\right)
$$

where the notation $\delta(\cdot)$ indicates the Dirac delta function and the sums go on all possible $k$ and on all possible sequences. The support of this density is in $\left[\epsilon_{0}=-1 / 2, \epsilon_{\max }=1\right]$.

Accordingly, the average energy is

$$
\int_{\epsilon_{0}}^{1} d \epsilon \rho(\epsilon) \epsilon=\sum_{k=0}^{\infty}(1 / 3)^{k+1} \sum_{\bar{\sigma}_{k}} \epsilon\left(k, \bar{\sigma}_{k}\right)=0
$$

where the second equality can be simply demonstrated using iteratively 8 with the initial condition $\epsilon(0)=0$, which immediately gives $\sum_{\bar{\sigma}_{k}} \epsilon\left(k, \bar{\sigma}_{k}\right)=0$ for any $k$.

The cumulative distribution of states can be obtained by the integration of the density of states:

$$
P(\epsilon)=\int_{\epsilon_{0}}^{\epsilon} d \epsilon^{\prime} \rho\left(\epsilon^{\prime}\right)=\sum_{k=0}^{\infty}(1 / 3)^{k+1} \sum_{\bar{\sigma}_{k}} \theta\left(\epsilon-\epsilon\left(k, \bar{\sigma}_{k}\right)\right)
$$

where $\theta(x)$ is the step function $(\theta(x)=0$ for $x<0$ and $\theta(x)=1$ for $x \geq 0)$. The density of states $\rho(\epsilon)$ is normalized since, as it is easy to verify, $P(1)=1$. This cumulative distribution depicted in Fig. 2 is generated by a $g=20$ network $\left(N_{g}=6,973,568,804\right)$. The fractal nature of its support is evident, since there are steps of all sizes. Moreover, it can be seen that close to extremes it presents a self-similar structure that will be better described in the following.

Let us see more in detail the behavior of the cumulative distribution close to the two extremes $\epsilon_{\max }=1$ and $\epsilon_{0}=-1 / 2$. Let us start by rewriting 11 as

$$
P(\epsilon)=\frac{1}{3} \theta(\epsilon)+\sum_{k=0}^{\infty}(1 / 3)^{k+2} \sum_{\bar{\sigma}_{k}} \sum_{\sigma_{k+1}} \theta\left(\epsilon-\epsilon\left(k+1, \bar{\sigma}_{k+1}\right)\right),
$$

where $\sigma_{k+1}$ in the last sum takes the two possible values \pm 1 . Using 8 one gets

$$
\sum_{\sigma_{k+1}} \theta\left(\epsilon-\epsilon\left(k+1, \bar{\sigma}_{k+1}\right)\right)=1+\left[2 \theta\left(4 \epsilon-\epsilon\left(k, \bar{\sigma}_{k}\right)\right)-1\right] \theta\left(q(\epsilon)-\epsilon\left(k, \bar{\sigma}_{k}\right)\right),
$$

where $q(\epsilon)=\left(6 \epsilon^{2}-1\right) /(3 \epsilon+2)$. When $\epsilon>1 / 4$, since all $\epsilon\left(k, \bar{\sigma}_{k}\right)$ are equal or smaller than unity, one has $\theta(4 \epsilon-$ $\left.\epsilon\left(k, \bar{\sigma}_{k}\right)\right)=1$. Then, for $\epsilon>1 / 4$, expression 13 has the simpler form:

$$
\sum_{\sigma_{k+1}} \theta\left(\epsilon-\epsilon\left(k+1, \bar{\sigma}_{k+1}\right)\right)=1+\theta\left(q(\epsilon)-\epsilon\left(k, \bar{\sigma}_{k}\right)\right)
$$

so that for $\epsilon>1 / 4$ equation 12 gives

$$
P(\epsilon)=\frac{2}{3}+\frac{1}{3} P(q(\epsilon))
$$

For $\epsilon$ close to $\epsilon_{\max }=1$, using $\epsilon=1-x$, the linearization of $q(\epsilon)$ gives $q(\epsilon)=q(1-x) \simeq 1-(9 / 5) x$, then after having defined $Q(x)=1-P(1-x)$ we obtain

$$
Q(x) \simeq \frac{1}{3} Q\left(\frac{9}{5} x\right)
$$


which for any value of $x$ gives rise to sequences of points geometrically spaced corresponding to a geometrically valued cumulative distribution. There are two consequences, the first one being that close to $\epsilon_{\max }=1$ the cumulative distribution is self-similar, the second is that any of the sequence of points must be on a geometrical curve $a x^{1+\delta}$ which must satisfy 16$)$, which implies $1+\delta=\ln (3) / \ln (9 / 5) \approx 1.869$.

When $\epsilon<-1 / 8$, since all $\epsilon\left(k, \bar{\sigma}_{k}\right)$ are equal or larger than $\epsilon_{0}=-1 / 2$, one has $\theta\left(4 \epsilon-\epsilon\left(k, \bar{\sigma}_{k}\right)\right)=0$. Then, for $\epsilon>1 / 4$, we have from 13

$$
\sum_{\sigma_{k+1}} \theta\left(\epsilon-\epsilon\left(k+1, \bar{\sigma}_{k+1}\right)\right)=1-\theta\left(q(\epsilon)-\epsilon\left(k, \bar{\sigma}_{k}\right)\right),
$$

so that for $\epsilon<-1 / 8$ equation 12 gives

$$
P(\epsilon)=\frac{1}{3}-\frac{1}{3} P(q(\epsilon)) .
$$

For $\epsilon$ close to $\epsilon_{0}=-1 / 2$, using $\epsilon=x+\epsilon_{0}$, the linearization of $q(\epsilon)$ gives $q\left(x+\epsilon_{0}\right)=1-18 x$. Therefore

$$
P\left(x+\epsilon_{0}\right)=\frac{1}{3}[1-P(1-18 x)]=\frac{1}{3} Q(18 x)
$$

so that the self-similar structure of $Q(x)$ is entirely reflected in $P\left(x+\epsilon_{0}\right)$, whose corresponding sequences of points are on geometrical curves $b\left(\epsilon-\epsilon_{0}\right)^{1+\delta}$.

This can be appreciated in Fig. 3 which shows the self-similar cumulative distribution near the band bottom $\epsilon_{0}=-1 / 2$; in particular it is shown that the two envelopes which contain $P(\epsilon)$ and which correspond to two distinct sequences of points generated by 1619 behave proportionally to $\left(\epsilon-\epsilon_{0}\right)^{1+\delta}$ with $1+\delta=\ln (3) / \ln (9 / 5) \approx 1.869$. In other words

$$
b_{-}\left(\epsilon-\epsilon_{0}\right)^{1+\delta} \leq P(\epsilon) \leq b_{+}\left(\epsilon-\epsilon_{0}\right)^{1+\delta}
$$

with $b_{-} \approx 19.0$ and $b_{+} \approx 38.6$.

This super-linear behavior of the cumulative distribution of states close to the band bottom points to a BEC transition at finite temperature, as it will be confirmed in next section.

The exponent $\delta$ corresponds to the spectral dimension $d_{s}=2(1+\delta) \approx 3.738$ associated to the generator of the process with jump rates $h_{i, j}$ between connected nodes. Notice that the spectral dimension has a value larger then 2 while the embedding dimension of the network has dimension 2.

\section{THERMODYNAMICS.}

Let us now consider a gas of $N_{p}$ non interacting bosons which can occupy any of the eigenstates corresponding to the $N_{g}$ eigenvalues of the Hamiltonian. The average number of bosons in each eigenstate is, therefore, $N_{p} / N_{g}$ which we name bosonic density. In the thermodynamic limit $N_{p}$ diverges while $N_{g}$ diverges in the infinite network size limit. In order to have a non trivial thermodynamics, it is compulsory to assume that the bosonic density remains finite and strictly positive. In other words, we assume that in the limit where both $N_{p}$ and $N_{g}$ diverge one has $N_{p} / N_{g} \rightarrow \phi$ with $0<\phi<\infty$.

We also define $\phi_{0}$ as the density of condensed bosons (number of bosons in the ground state divided by the number of eigenstates) and $\phi_{t h}$ as the density of thermal bosons (number of bosons not in the ground state divided by the number of eigenstates). Then one trivially has $\phi=\phi_{0}+\phi_{t h}$.

We can then work directly in the thermodynamic limit (see [9]) and write

$$
\phi=\phi_{0}+\phi_{t h}=\phi_{0}+\int_{\epsilon_{0}}^{1} d \epsilon \rho(\epsilon) \frac{1}{z^{-1} e^{\beta\left(\epsilon-\epsilon_{0}\right)}-1},
$$

where $\beta=1 / T$ is the inverse temperature (units with Boltzmann constant $K_{B}=1$ ) and $z$ is the fugacity.

For $T<T_{c}$ one has $z=1$ and $\phi_{0}(\phi, T)=\phi-\phi_{t h}(T)$. Notice that, since $z=1, \phi_{t h}(T)$ only depends on temperature and not on $\phi$. For $T>T_{c}$ one has $z<1$ and $\phi_{0}=0$ which implies $\phi_{t h}=\phi$. When $\phi_{0}=0$ equation (21) gives $z(\phi, T)$ as implicit solution. 


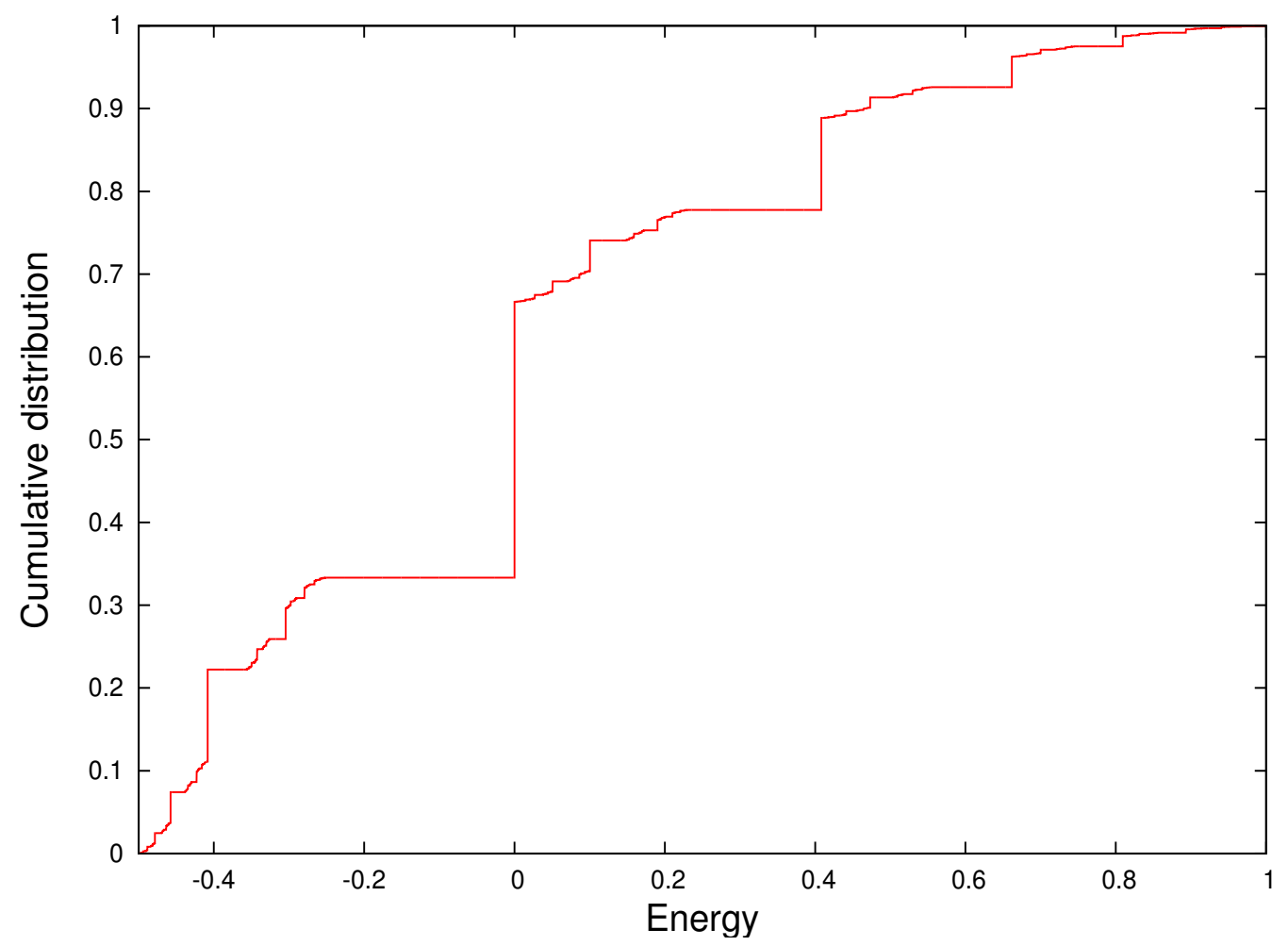

FIG. 2: Cumulative distribution of states $P(\epsilon)$. Degeneracies and gaps are signaled by vertical and horizontal segments, respectively. Close to the extremes $P(\epsilon)$ shows a self-similar structure. This figure has been obtained considering a $g=20$ network corresponding to $N_{g}=6,973,568,804$ nodes.

\section{A. Critical temperature.}

The critical temperature $T_{c}(\phi)=1 / \beta_{c}(\phi)$ is found as solution of 21) setting both $z=1$ and $\phi_{0}=0$ :

$$
\phi=\int_{\epsilon_{0}}^{1} d \epsilon \rho(\epsilon) \frac{1}{e^{\beta_{c}\left(\epsilon-\epsilon_{0}\right)}-1},
$$

which is depicted in Fig. 4 in a log-log plot showing two different power-law regimes that can be deduced from (22).

When $T_{c}$ is small ( $\beta_{c}$ is large) only values of $\epsilon$ close to $\epsilon_{0}$ contribute to the integral. Thus, taking into account that for small values of $\epsilon$ one has $P(\epsilon)=\int_{\epsilon_{0}}^{\epsilon} d \epsilon^{\prime} \rho\left(\epsilon^{\prime}\right) \sim\left(\epsilon-\epsilon_{0}\right)^{1+\delta}$, one gets

$$
\phi \sim \int_{\epsilon_{0}}^{1} d \epsilon \frac{\left(\epsilon-\epsilon_{0}\right)^{\delta}}{e^{\beta_{c}\left(\epsilon-\epsilon_{0}\right)}-1}=T_{c}^{1+\delta} \int_{0}^{\frac{3}{2} \beta_{c}} d \epsilon \frac{x^{\delta}}{e^{x}-1},
$$

and finally, neglecting terms exponentially small in $\beta_{c}$, one gets

$$
T_{c} \simeq a_{-} \phi^{\frac{1}{1+\delta}},
$$

where $a_{-}$is numerically determined to be $a_{-} \approx 0.1$.

On the contrary, when $T_{c}$ is large $\left(\beta_{c}\right.$ is small) one has $e^{\beta_{c}\left(\epsilon-\epsilon_{0}\right)}-1 \simeq \beta_{c}\left(\epsilon-\epsilon_{0}\right)$ and straightforwardly from 22 we derive

$$
T_{c} \simeq a_{+} \phi,
$$

where $a_{+}=1 / \int_{\epsilon_{0}}^{1} d \epsilon \rho(\epsilon) \frac{1}{\epsilon-\epsilon_{0}} \approx 0.15$. These two regimes are confirmed by plot in Fig.4. 


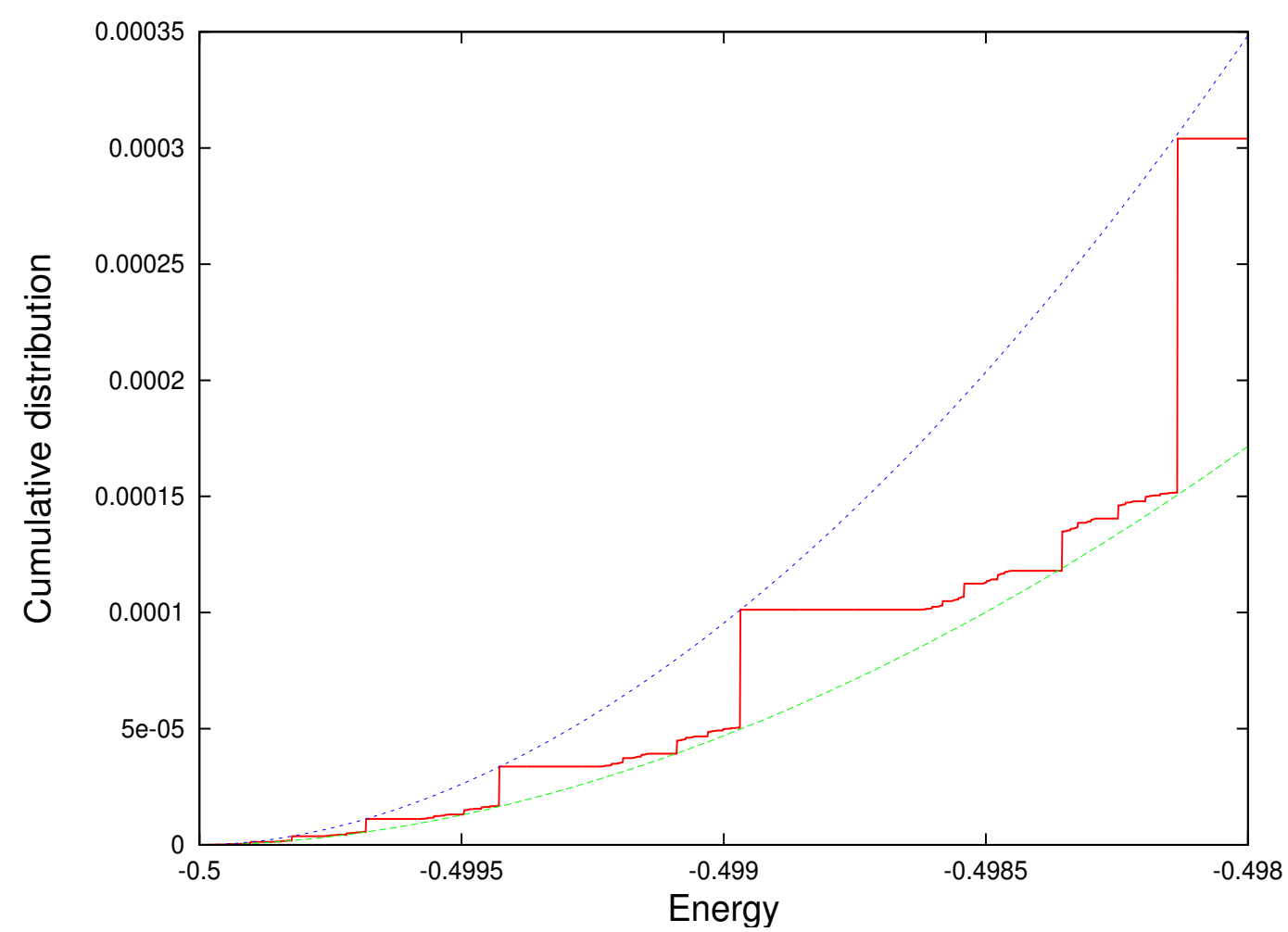

FIG. 3: Cumulative distribution of states $P(\epsilon)$ close to the band bottom plotted together with the two envelopes $b_{-}(\epsilon+0.5)^{1+\delta}$ and $b_{+}(\epsilon+0.5)^{1+\delta}\left(b_{-} \approx 19.0\right.$ and $\left.b_{+} \approx 38.6\right)$. This super-linear behavior of the cumulative distribution close to the band bottom, corresponding to the spectral dimension $d_{s}=2(1+\delta) \approx 3.738$, points to a BEC transition at finite temperature.

\section{B. Bose-Einstein condensate.}

For $T<T_{c}$ the condensed fraction $f_{0}(\phi, T)=\phi_{0} / \phi$ is

$$
f_{0}(\phi, T)=1-\frac{\phi_{t h}(T)}{\phi}=1-\frac{1}{\phi} \int_{\epsilon_{0}}^{1} d \epsilon \rho(\epsilon) \frac{1}{e^{\beta\left(\epsilon-\epsilon_{0}\right)}-1}
$$

which is depicted in Figs 5, 6 and vanishes for $T=T_{c}$ where $\phi_{t h}=\phi$.

Using exactly the same argument as above one obtains that for small temperature $T$ (large $\beta$ ) one has $\phi_{t h} \sim T^{1+\delta}$ while for large temperature $T$ (small $\beta$ ) one has $\phi_{t h} \sim T$. Therefore, since a small $\phi$ implies a small $T_{c}$ and since $f_{0}(\phi, T)$ is non vanishing only when $T<T_{c}$ one has

$$
f_{0}(\phi, T) \simeq 1-\left(\frac{T}{T_{c}}\right)^{1+\delta}
$$

which holds for small $\phi$ and where $T_{c}$ depends on $\phi$ according to (22) approximated by (24) (see Fig. 5).

On the contrary, a large $\phi$ implies a large $T_{c}$ and since $f_{0}(\phi, T)$ is non vanishing in all region $T<T_{c}$ one has that the previous relation only holds for $T$ close to 0 while for larger $T$ one has

$$
f_{0}(\phi, T) \simeq 1-\left(\frac{T}{T_{c}}\right),
$$

which holds for large $\phi$ and where the critical temperature $T_{c}$ depends on $\phi$ according to (22) approximated by (25) (see Fig. 6).

Interestingly, for small densities, the behavior is determined by the bottom of energy band and it corresponds to a free boson gas in dimension $d_{s}=2(1+\delta) \approx 3.738\left(d_{s}\right.$ is the spectral dimension). 


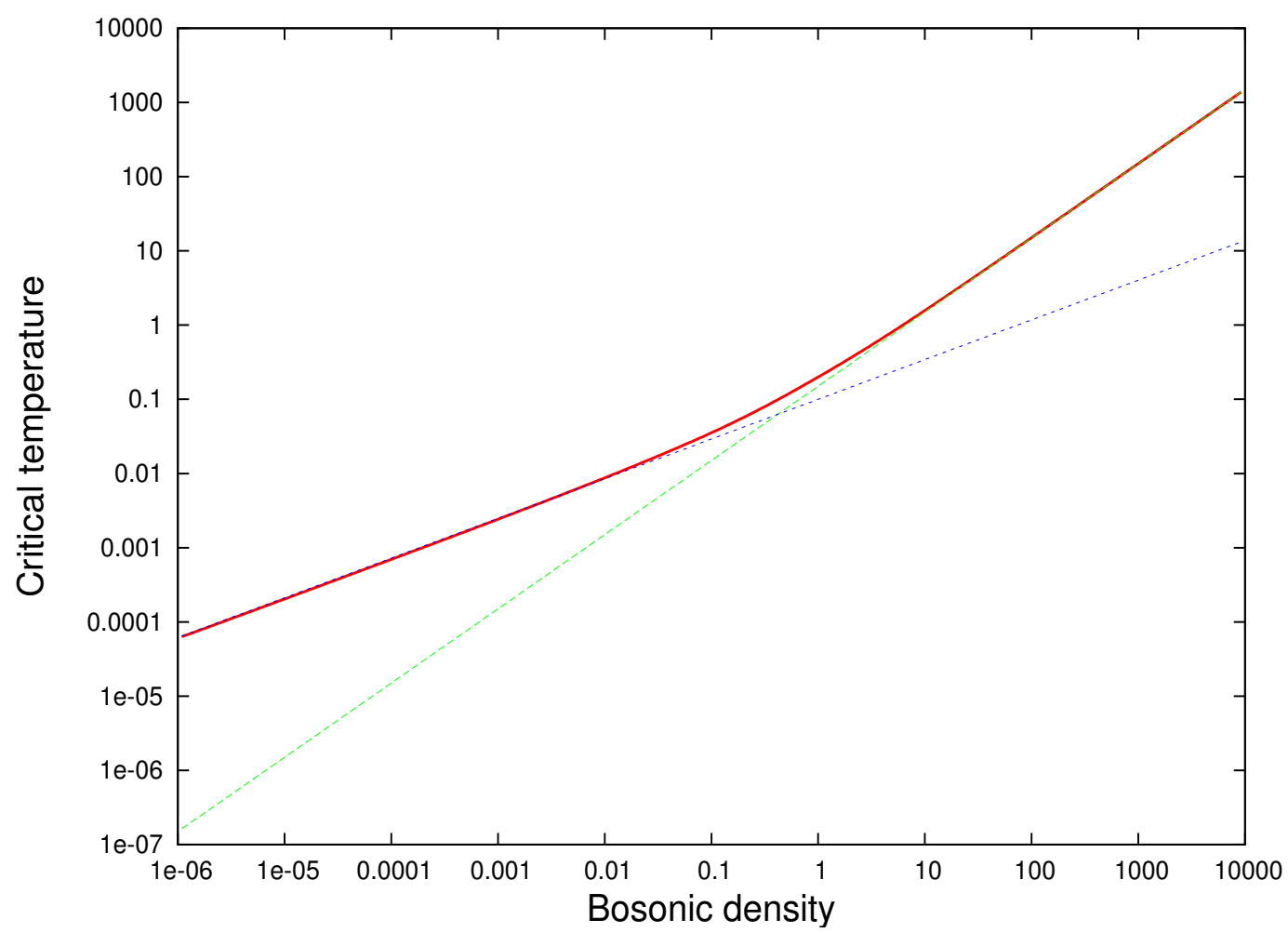

FIG. 4: Critical temperature $T_{c}$ versus bosonic density $\phi$ compared with $0.1 \phi^{1 /(1+\delta)}$ (small bosonic density) and $0.15 \phi$ (large bosonic density).

\section{Fugacity.}

The fugacity $z(\phi, T)$ equals 1 for $T<T_{c}$ while for $T>T_{c}$ can be obtained from 21 setting $\phi_{0}=0$ which gives

$$
\phi=\int_{\epsilon_{0}}^{1} d \epsilon \rho(\epsilon) \frac{1}{z^{-1} e^{\beta\left(\epsilon-\epsilon_{0}\right)}-1},
$$

and, in particular, when $\beta=0(T \rightarrow \infty)$ one has $\phi=1 /\left(z^{-1}-1\right)$ which gives $\lim _{T \rightarrow \infty} z(\phi, T)=\phi /(\phi+1)$.

Then, from (21), keeping $\phi$ constant and deriving with respect to the inverse temperature, one gets

$$
\frac{\partial z}{\partial \beta}=\frac{z \int_{\epsilon_{0}}^{1} d \epsilon \rho(\epsilon) \frac{\left(\epsilon-\epsilon_{0}\right) e^{\beta\left(\epsilon-\epsilon_{0}\right)}}{\left[z^{-1} e^{\beta\left(\epsilon-\epsilon_{0}\right)}-1\right]^{2}}}{\int_{\epsilon_{0}}^{1} d \epsilon \rho(\epsilon) \frac{e^{\beta\left(\epsilon-\epsilon_{0}\right)}}{\left[z^{-1} e^{\beta\left(\epsilon-\epsilon_{0}\right)}-1\right]^{2}}} .
$$

When $z=1$, the integrand in the numerator of right-hand side of 30 behaves as $\rho(\epsilon)\left(\epsilon-\epsilon_{0}\right)^{-1}$ while the integrand in the denominator as $\rho(\epsilon)\left(\epsilon-\epsilon_{0}\right)^{-2}$. Since $P(\epsilon)=\int_{\epsilon_{0}}^{\epsilon} d \epsilon^{\prime} \rho\left(\epsilon^{\prime}\right) \sim\left(\epsilon-\epsilon_{0}\right)^{1+\delta} \approx\left(\epsilon-\epsilon_{0}\right)^{1.869}$, the numerator is finite

while the denominator diverges, so that equation $\sqrt{30}$, when $z=1$ (when $T \leq T_{c}$ ), gives $\frac{\partial z}{\partial \beta}=0$. Therefore, equation (30) can be used to operatively compute $z$ for all temperatures given that at $\beta=0$ one has $z=\phi /(\phi+1)$. The result is shown in Fig. 7 where $z(\phi, T)$ is plotted against the rescaled temperature $T / T_{c}$ for various values of $\phi$.

\section{Energy and specific heat}

The energy density $E(\phi, T)$ (thermodynamic limit of the ratio between the total energy and number of eigenstates) is given by

$$
E=\int_{\epsilon_{0}}^{1} d \epsilon \rho(\epsilon) \frac{\epsilon}{z^{-1} e^{\beta\left(\epsilon-\epsilon_{0}\right)}-1}+\epsilon_{0} \phi_{0}=\int_{\epsilon_{0}}^{1} d \epsilon \rho(\epsilon) \frac{\epsilon-\epsilon_{0}}{z^{-1} e^{\beta\left(\epsilon-\epsilon_{0}\right)}-1}+\epsilon_{0} \phi
$$




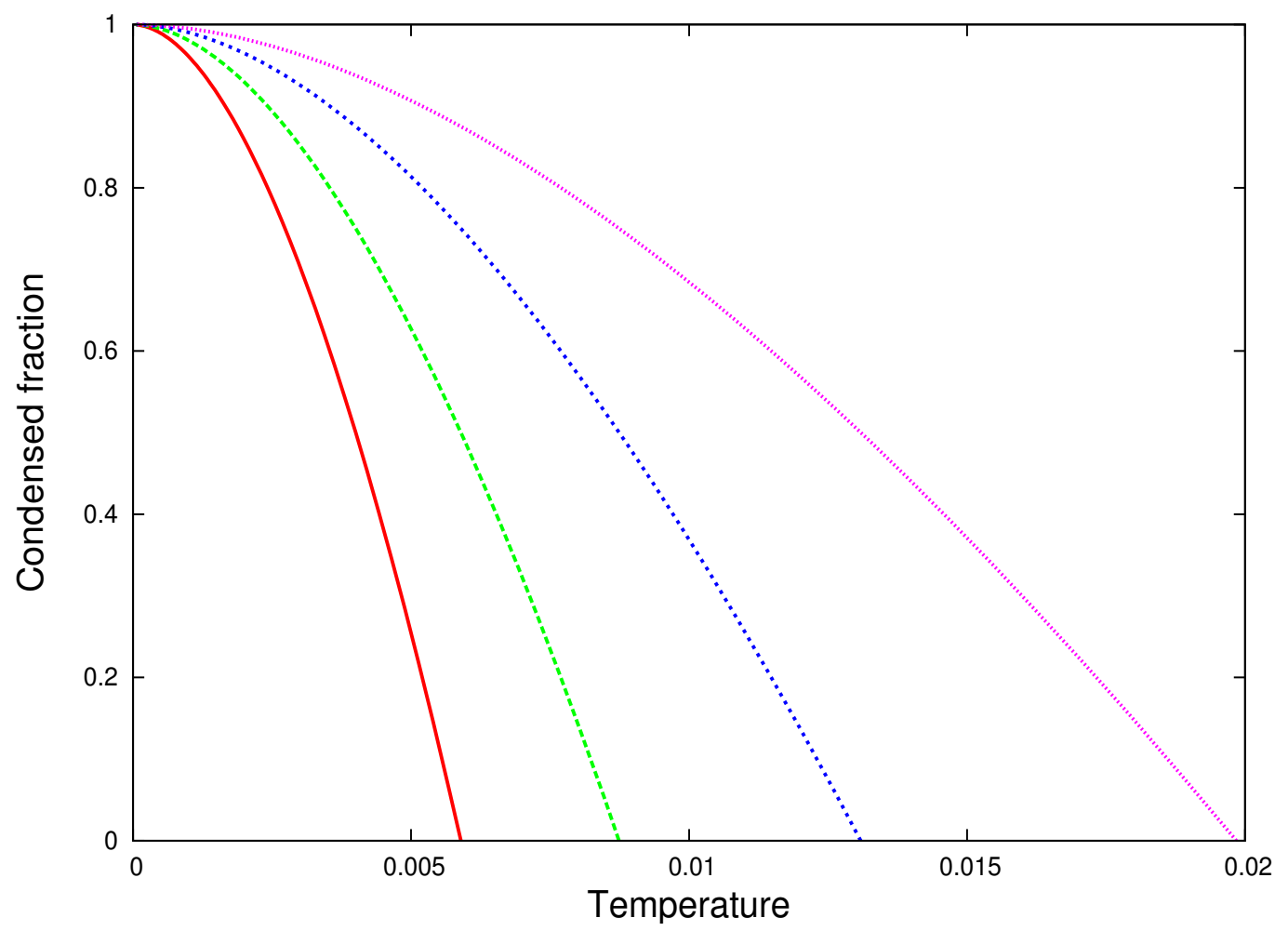

FIG. 5: Condensed fraction $f_{0}(\phi, T)$ versus temperature $T$ for $\phi=0.005, \phi=0.01, \phi=0.02, \phi=0.04$ (from left to right). For these small densities, the behavior corresponds to a free boson gas in dimension $d_{s}=2(1+\delta) \approx 3.738\left(d_{s}\right.$ is the spectral dimension of the model). One has $f_{0}(\phi, T) \simeq 1-\left(\frac{T}{T_{c}}\right)^{1+\delta}$.

In the $T \rightarrow \infty$ limit one has that $z=\phi /(1+\phi)$ therefore

$$
\lim _{T \rightarrow \infty} E=\phi \int_{\epsilon_{0}}^{1} d \epsilon \rho(\epsilon) \epsilon=0,
$$

where the second equality was proven in Section 4 . On the other hand, in the limit $T \rightarrow 0$. as it is obvious, one has $E \rightarrow \epsilon_{0} \phi=-\phi / 2$.

The specific heat density $C(\phi, T)$ is obtained by deriving (31) with respect to the temperature keeping the bosonic density fixed.

$$
C=\frac{1}{z^{2} T^{2}} \int_{\epsilon_{0}}^{1} d \epsilon \rho(\epsilon) \frac{z\left(\epsilon-\epsilon_{0}\right)-\frac{\partial z}{\partial \beta}}{\left[z^{-1} e^{\beta\left(\epsilon-\epsilon_{0}\right)}-1\right]^{2}}\left(\epsilon-\epsilon_{0}\right) e^{\beta\left(\epsilon-\epsilon_{0}\right)},
$$

where $\frac{\partial z}{\partial \beta}$, given by equation 30 vanishes for $T \leq T_{c}$.

The specific heat below the critical temperature is independent on $\phi$ since $z=1$ and $\frac{\partial z}{\partial \beta}=0$. This fact can be appreciated in Figs. 8 and 9 (notice that the four curves corresponding to different values of $\phi$ are in two different figures because the involved scale of temperature is different). At the critical temperature the specific heat has a cusp, with a finite derivative at the left side and infinite derivative at the right side as it is depicted both in Fig. 8 and in Fig. 9.

\section{DISCUSSION}

The main results out of this paper are:

(i) the exact computation of the eigenvalues of a tight-binding Hamiltonian on the RAN substrate for any finite size of the network; 


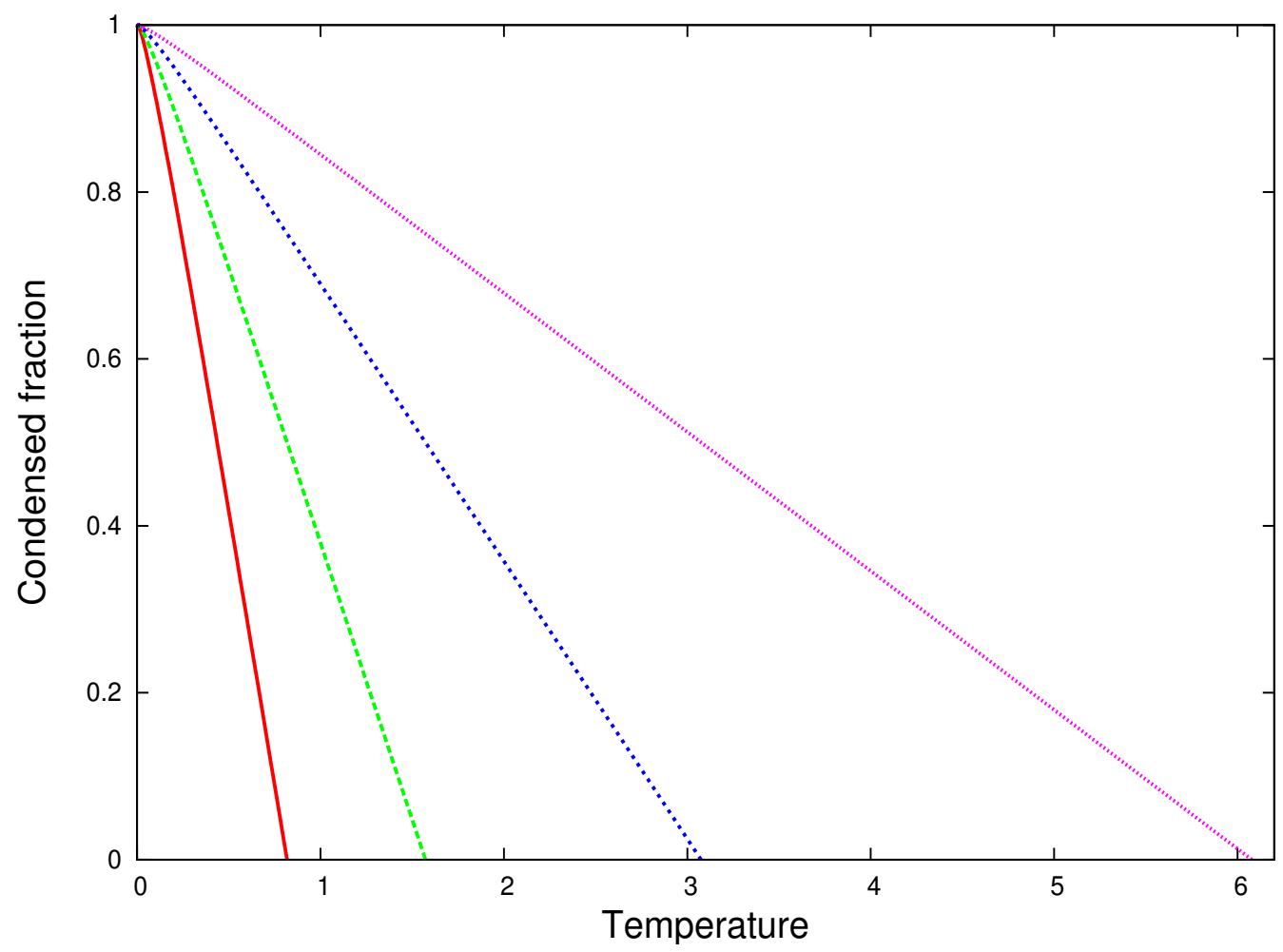

FIG. 6: Condensed fraction $f_{0}(\phi, T)$ versus temperature $T$ for $\phi=5, \phi=10, \phi=20, \phi=40$ (from left to right). For these large densities, the behavior is determined by the fact that there is a finite maximum eigenvalue of the Hamiltonian. One has $f_{0}(\phi, T) \simeq 1-\left(\frac{T}{T_{c}}\right)$.

(ii) the exact computation of the cumulative density of states of this Hamiltonian in the limit of infinite size network for which we also described the properties in the relevant region close to the ground state energy;

(iii) the proof of BEC and its complete description in terms of transition line, fraction of condensed particles, fugacity, energy and specific heat.

Since RAN is embedded in a two dimensional space, this result add more evidence to the catalyst role of topology for the existence of a Bose-Einstein condensate. The relevant parameter for the existence of the condensate is the spectral dimension $d_{s}$ which has to be larger then 2. Since the spectral dimension $d_{s}$ and the physical dimension $d$ coincide for translationally invariant lattices, there is not contradiction with the prescription $d>2$ for these models. In this present model, on the contrary, we have $d_{s} \approx 3.738>d=2$ which explains how the topology may induce condensation.

\section{Acknowledgments}

The author thanks Eudenilson Lins Albuquerque, Umberto Laino Fulco, Marcelo Leite Lyra and Pasquale Sodano for discussions, suggestions and a critical reading of the manuscript. Financial support from the Brazilian Research Agencies CAPES (Rede NanoBioTec and PNPD), CNPq [INCT-Nano(Bio) Simes, Casadinho-Procad] and FAPERN/CNPq (PRONEM) and PRIN 2009 protocollo n. 2009TA2595.02 is also acknowledged.

[1] Anderson M H, Ensher J R, Matthews M R, Wieman C E and Cornell E A, Observation of Bose-Einstein condensation in a dilute atomic vapor, Science 269, 198 (1995)

[2] Davis K B, Mewes M-O, Andrews M R, van Druten N J, Durfee D S, Kurn D M and Ketterle W, Bose-Einstein condensation in a gas of sodium atoms, Phys. Rev. Lett. 75, 3969 (1995) 


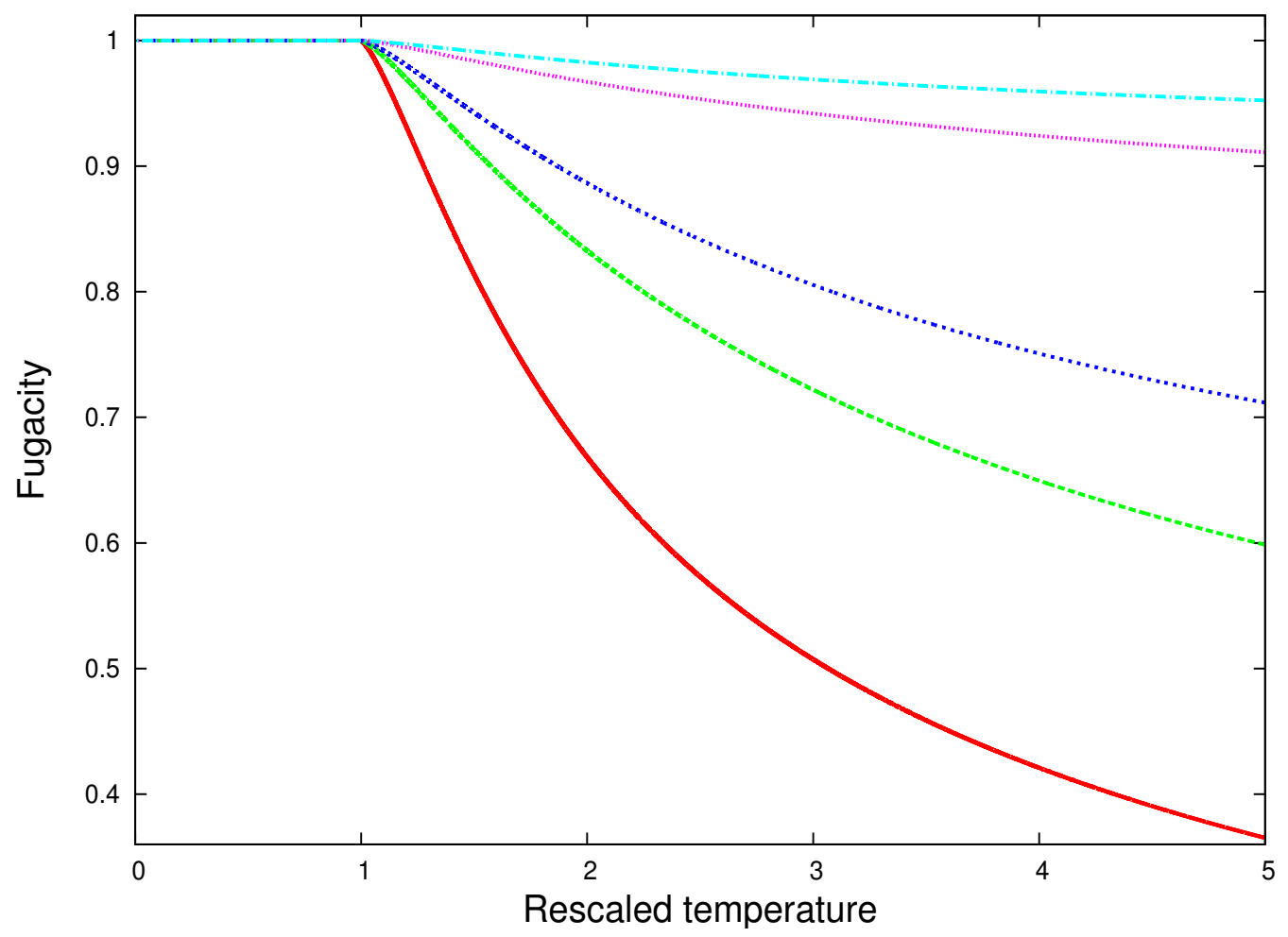

FIG. 7: The fugacity $z(\phi, T)$ versus the rescaled temperature $T / T_{c}$ for $\phi=.1, \phi=.5, \phi=1, \phi=5$ and $\phi=10$ (from below). The asymptotic value for $T \rightarrow \infty$ is $z=\phi /(\phi+1)$. The fugacity behaves linearly in the proximity of the transition.

[3] Butov L W, Lai C W, Ivanov A L, Gossard A C and Chemla D S, Towards Bose-Einstein condensation of excitons in potential traps, Nature 417, 47 (2002)

[4] Kasprzak J, Richard M, Kundermann S , Baas A, Jeambrun P, Keeling J M J, Marchetti F M, Szymańska M H, André R, Staehli J L, Savona V, Littlewood B P, Deveaud B and Le Si Dang, Bose-Einstein condensation of exciton polaritons, Nature 443, 409 (2006)

[5] Demokritov S O, Demidov V E, Dzyapko O, Melkov G A, Serga A A, Hillebrands B and Slavin A N, Bose-Einstein condensation of quasi-equilibrium magnons at room temperature under pumping, Nature 443, 430 (2006)

[6] Giamarchi T, Rüegg C and Tchernyshyov 0, Bose-Einstein condensation in magnetic insulators, Nature Physics 4, 198 (2008)

[7] Plumhof J D, Stöferle T, Mai L, Scherf U and Mahrt R F, Room-temperature BoseEinstein condensation of cavity excitonpolaritons in a polymer, Nature Materials doi:10.1038/nmat3825 (2013)

[8] Burioni R, Cassi D, Meccoli I, Rasetti M, Regina S, Sodano P and Vezzani A, Bose-Einstein condensation in inhomogeneous Josephson arrays, Europhys. Lett. 52, 251 (2000)

[9] Burioni R, Cassi D, Rasetti M, Sodano P and Vezzani A, Bose-Einstein condensation on inhomogeneous complex networks, J. Phys. B: At. Mol. Opt. Phys. 34, 4697 (2001)

[10] Buonsante P, Burioni R, Cassi D and Vezzani A, Bose-Einstein condensation on inhomogeneous networks: Mesoscopic aspects versus thermodynamic limit, Phys. Rev. B 66, 094207 (2002)

[11] Brunelli I, Giusiano G, Mancini F P, Sodano P and Trombettoni A, Topology-induced spatial Bose-Einstein condensation for bosons on star-shaped optical network, J. Phys. B: At. Mol. Opt. Phys. 37, 275 (2004)

[12] Sodano P, Trombettoni A, Silvestrini P, Russo R, and Ruggiero B, Inhomogeneous superconductivity in comb-shaped Josephson junction networks, New J. Phys. 8, 327 (2006)

[13] Vidal E J G G, Lima R P A and Lyra M L, Bose-Einstein condensation in the infinitely ramified star and wheel graphs, Phys. Rev. E 83, 061137 (2011)

[14] Cardoso A L, Andrade R F S and Souza A M C, Localization properties of a tight-binding electronic model on the Apollonian network, Phys. Rev. B 78, 214202 (2008)

[15] de Oliveira I N, de Moura F A B F, Lyra M L, Andrade Jr J S and Albuquerque E L, Free-electron gas in the Apollonian network: Multifractal energy spectrum and its thermodynamic fingerprints, Phys. Rev. E 79, 016104 (2009)

[16] de Oliveira I N, de Moura F A B F, Lyra M L, Andrade Jr J S and Albuquerque E L, Bose-Einstein condensation in the Apollonian complex network, Phys. Rev. E 81, 030104(R) (2010)

[17] de Oliveira I N, dos Santos T B, de Moura F A B F, Lyra M L and Serva M, Critical behavior of the ideal-gas Bose-Einstein condensation in the Apollonian network, Phys. Rev. E 88, 022139 (2013) 


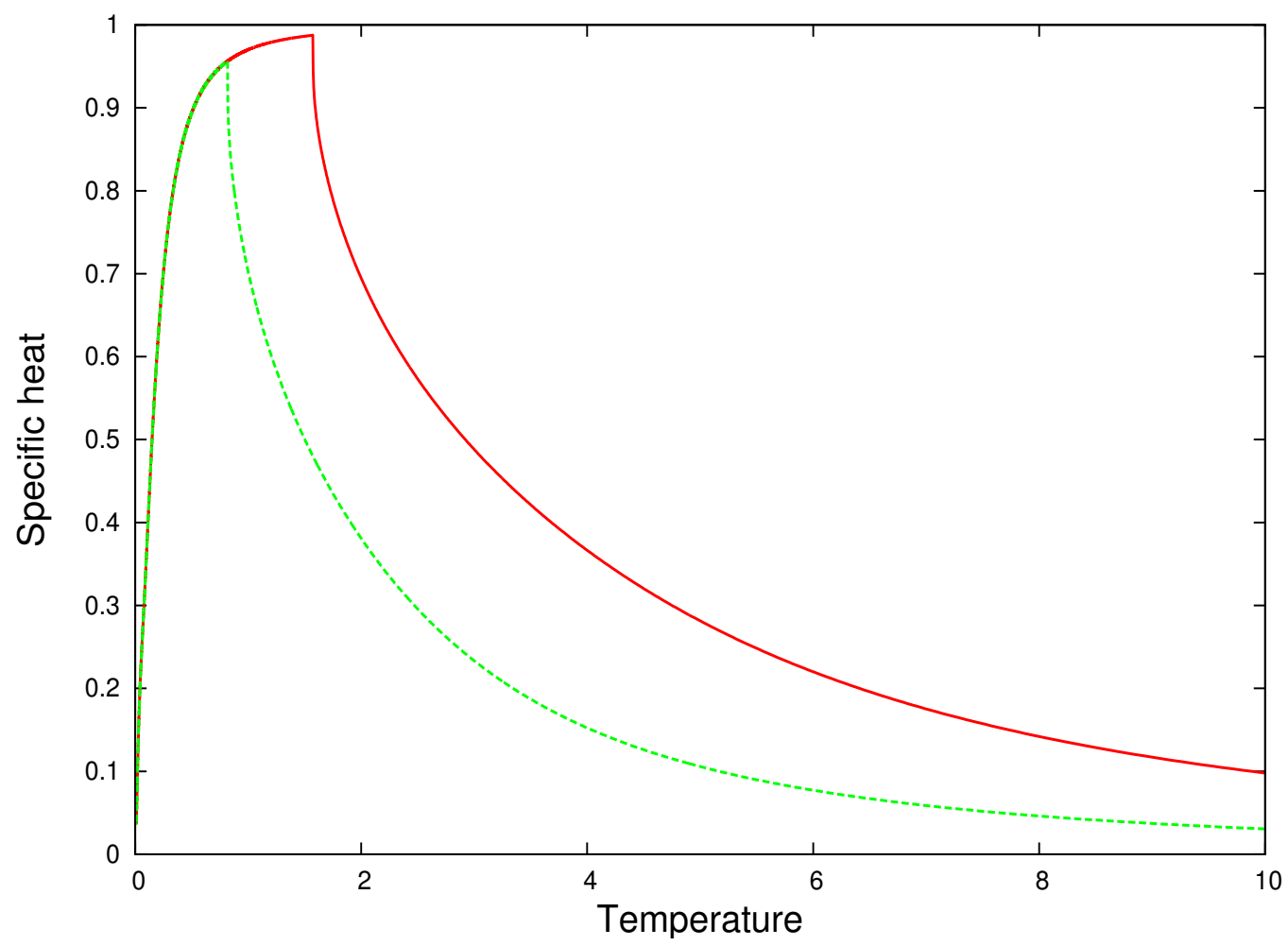

FIG. 8: Specific heat $C$ versus temperature $T$ for $\phi=5$ (green, dashed line) and $\phi=10$ (red, full line). The specific heat below the critical temperature is independent on $\phi$ as it is depicted in the figure. At the critical temperature $C$ has a cusp, with a finite derivative at the left side and infinite derivative at the right side.

[18] Serva M, Fulco U L and Albuquerque E L, Ising models on the regularized Apollonian network, Phys. Rev. E 88, 042823 (2013)

[19] Serva M, Fulco U L and Albuquerque E L, Exact solutions and infinite-order phase transitions for a general class of Ising models on the regularized Apollonian network, J. Stat. Mech. P01010, (2014)

[20] Andrade Jr J S, Herrmann H J, Andrade R F S and da Silva L R, Apollonian Networks: Simultaneously Scale-Free, Small World, Euclidean, Space Filling, and with Matching Graphs, Phys. Rev. Lett. 94, 018702 (2005)

[21] da Silva L F, Costa Filho R N, Soares D J B, Fulco U L and Albuquerque E L, Critical properties of contact process on the Apollonian network, Physica A 392, 1532 (2013)

[22] da Silva L F, Costa Filho R N, Cunha A R, Macedo-Filho A, Serva M, Fulco U L and Albuquerque E L, Critical properties of the SIS model dynamics on the Apollonian network, J. Stat. Mech. P05003 (2013)

[23] Lima F W S, Moreira A A and Araújo A D, Nonequilibrium model on Apollonian networks. Phys. Rev. E 86, 056109 (2012)

[24] Andrade R F S and Herrmann H J, Magnetic models on Apollonian networks, Phys. Rev. E 71, 056131 (2005)

[25] Andrade R F S, Andrade Jr J S and Herrmann H J, Ising model on the Apollonian network with node-dependent interactions, Phys. Rev. E 79, 036105 (2009)

[26] Araújo N A M, Andrade R F S and Herrmann H J, q-state Potts model on the Apollonian network, Phys. Rev. E 82, 046109 (2010)

[27] Doye J P K and Massen C P, Self-similar disk packings as model spatial scale-free networks, Phys. Rev. E 71, 016128 (2005)

[28] Lind P G, da Silva L R, Andrade Jr J S and Herrmann H J, Spreading gossip in social networks, Phys. Rev. E 76, 036117 (2007)

[29] Baxter R J, Exactly solved models in statistical mechanics, (Academic Press, London, 1982)

[30] Georges A, Kotliar G, Krauth W and Rozenberg M J, Dynamical mean-field theory of strongly correlated fermion systems and the limit of infinite dimensions, Rev. Mod. Phys. 68, 13 (1996)

[31] Antoni M and Ruffo S, Clustering and relaxation in Hamiltonian long-range dynamics, Phys. Rev. E 52, 2361 (1995).

[32] Serva M, Magnetization densities as replica parameters: The dilute ferromagnet, Phys. A 389, 2700 (2010)

[33] Serva M, Exact and approximate solutions for the dilute Ising model, Phys. A 390, 2443 (2011) 


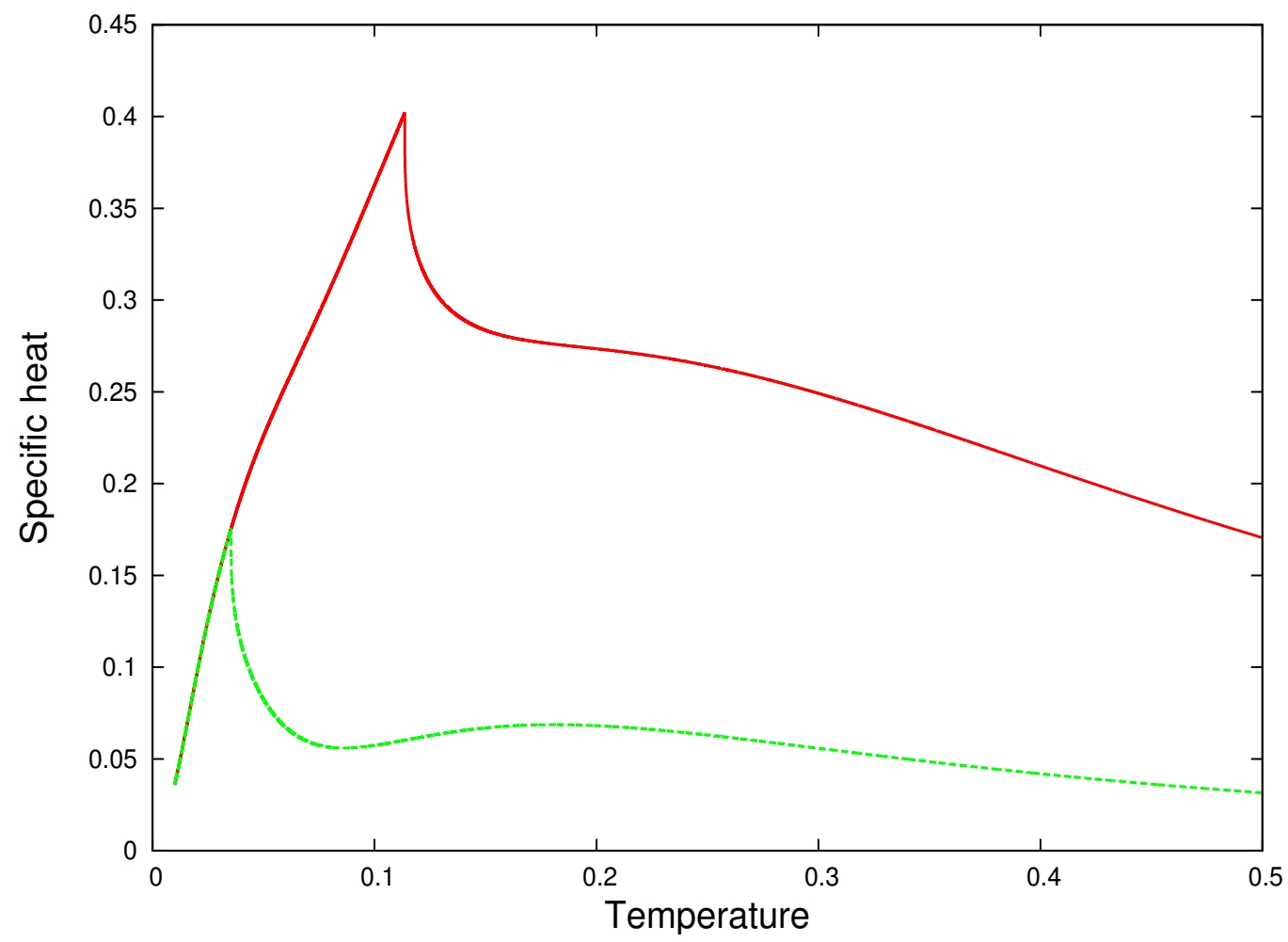

FIG. 9: Specific heat $C$ versus temperature $T$ for $\phi=.1$ (green, dashed line) and $\phi=.5$ (red, full line). Notice again that the specific heat below the critical temperature is independent on $\phi$, while at the critical temperature it has a cusp with a right side infinite derivative. 